\title{
Effect of Molecular Weight and Molar Ratio of Dextran on Self-Assembly of Dextran Stearate Polymeric Micelles as Nanocarriers for Etoposide
}

\author{
Jaleh Varshosaz, ${ }^{1}$ Farshid Hassanzadeh, ${ }^{2}$ Hojjat Sadeghi, ${ }^{1}$ \\ Farzin Firozian, ${ }^{3}$ and Mina Mirian ${ }^{3}$ \\ ${ }^{1}$ Department of Pharmaceutics, Faculty of Pharmacy and Novel Drug Delivery Systems Research Center, \\ Isfahan University of Medical Sciences, P.O. Box 81745-359, Isfahan 81746-73461, Iran \\ ${ }^{2}$ Department of Medicinal Chemistry, Faculty of Pharmacy, Isfahan University of Medical Sciences, Isfahan 81746-73461, Iran \\ ${ }^{3}$ Department of Biotechnology, Faculty of Pharmacy and Isfahan Pharmaceutical Sciences Research Center, \\ Isfahan University of Medical Sciences, Isfahan 81746-73461, Iran \\ Correspondence should be addressed to Jaleh Varshosaz, varshosaz@pharm.mui.ac.ir
}

Received 9 May 2012; Revised 21 September 2012; Accepted 18 October 2012

Academic Editor: Jun Liu

Copyright ( $\odot 2012$ Jaleh Varshosaz et al. This is an open access article distributed under the Creative Commons Attribution License, which permits unrestricted use, distribution, and reproduction in any medium, provided the original work is properly cited.

Amphiphilic polymer surfactants are composed of hydrophilic and hydrophobic polymers and are widely used in targeted drug delivery. The purpose of this study was the evaluation of the effect of molecular weight and molar ratio of dextran on physicochemical properties of dextran stearate polymeric micelles. Dextran stearate was synthesized by acylation of dextran with stearoyl chloride. Etoposide loaded polymeric micelles were prepared by dialysis method. The resulting micelles were evaluated for particle size, zeta potential, critical micelle concentration (CMC), drug loading capacity, and release efficiency. Cytotoxicity and cellular uptake of micelles were studied in CT-26 colorectal carcinoma cell line. Molecular weight and molar ratio of dextranstearate were impressive on zeta potential, CMC, drug loading capacity, and release efficiency. Unlike polymer molecular weight, molar ratio of stearate had a significant effect on cytotoxicity and particle size of etoposide loaded micelles. Although molecular weight of dextran had no significant effect on cytotoxicity of micelles on CT-26 cells, it had drastic attributes for stability of polymeric micelles. Consequently, both variables of molecular weight of dextran and molar ratio of stearate should be taken into account to have a stable and effective micelle of dextran-stearate.

\section{Introduction}

Polymeric micelles are self-assembling nanocarriers which are composed of two block copolymers. There are increasing interests in their use as drug carriers due to their stability and ease of preparation. They can solubilize, stabilize, and target pharmaceutical active ingredients and diagnostic materials $[1,2]$.

One of the most interesting areas of application of polymeric micelles is drug delivery to cancerous tumors. Many of chemotropic agents have low solubility and stability in blood or even during their storage time before administration. Most of them have severe adverse effects because of their distribution in other compartments of body than its target organ $[2,3]$.
When nanoparticles are administered via intravenous route and come into the tumors, they can pass across the fenestrated wall of the tumor capillaries, while they cannot pass away in normal tissues. This phenomenon is called enhanced permeation and retention (EPR) effect which allows passive targeting of pharmaceutical active ingredients to the tumors due to pathological abnormalities in the tumor vasculature $[4,5]$. Interendothelial gap defects increase vascular permeability in tumors, allowing extravasation of nanoparticles up to $400 \mathrm{~nm}$ [6].

Polymeric micelles composed from block copolymers show a core-shell micellar structure with a hydrophobic inner core surrounded by a hydrated outer shell in aqueous solution. Hydrophobic drugs can be incorporated in 
TABLE 1: Amounts of DMAP and stearoyl chloride needed for preparation of different dextran-stearate copolymers.

\begin{tabular}{lcccc}
\hline Dextran stearate copolymer & Dextran Mw & DMPA $(\mathrm{mg})$ & Stearoyl chloride $(\mu \mathrm{L})$ & Molar ratio of stearate/dextran \\
\hline $\mathrm{D}_{6} \mathrm{~S}_{7.5}$ & 6000 & 180 & $420(1.25 \mathrm{mmol})$ & 7.5 \\
$\mathrm{D}_{6} \mathrm{~S}_{10}$ & 6000 & 250 & $560(1.66 \mathrm{mmol})$ & 10 \\
$\mathrm{D}_{10} \mathrm{~S}_{7.5}$ & 10000 & 120 & $255(0.75 \mathrm{mmol})$ & 7.5 \\
$\mathrm{D}_{10} \mathrm{~S}_{10}$ & 10000 & 160 & $340(1 \mathrm{mmol})$ & 10 \\
\hline
\end{tabular}

the hydrophobic inner core and the hydrated outer shell helps to prevent opsonization of the carrier and uptake by the reticuloendothelial system. Polymeric micelles have the advantages of small particle size, smaller than $200 \mathrm{~nm}$ $[2,3]$. Polysaccharides are polymers of monosaccharides and are stable, nontoxic, safe, hydrophilic, and biodegradable materials used in pharmaceutical formulations [7].

Dextran is a branched polysaccharide composed of sucrose monomers. Addition of fatty acids to free- $\mathrm{OH}$ groups of dextran results in production of core-shell structure or amphiphilic polymeric micelle with surface active properties. For example, palmitoyl dextran is used as a nanocarrier for delivery of hirudin $[7,8]$.

Stearate grafted dextran had been synthesized by $\mathrm{Du}$ et al. [9] via an esterification reaction and was used to carry doxorubicin to nude mice bearing A549 human lung adenocarcinoma. In vitro cytotoxicity test showed good toxic effect against drug-sensitive tumor cells. Moreover, these micelles presented reversal activity against doxorubicinresistant cells.

Etoposide is a semisynthetic podophyllotoxin which act as an anticancer chemotropic agent with its topoisomerase II inhibitory effects. It has antitumor activity in a wide range of tumors including small-cell lung cancer (SCLC) and germ cell tumors of the testis and lymphomas. It has a low solubility in water and plasma half-life that can affect its clinical uses; so investigations on preparation of soluble, stable, and sustained release formulations of etoposide are continued [10].

Polymeric micelles of etoposide in poly- $\gamma$-benzyl- $l$ glutamate and poly(ethylene oxide) have been prepared and tested on CT-26 colorectal adenocarcinoma cell lines by MTT assay and tumor bearing BALB/c mice. Acceptable results have been obtained [11].

The main aim of this study was to prepare and optimize the polymeric micelles of etoposide by dextran stearate by studying the effect of dextran molecular weight on selfassembly of the micelles as nanocarriers for etoposide. Finally, these nanoparticles are evaluated in vitro and their cytotoxicity on colorectal cancer is studied on cell line of CT26.

\section{Materials and Methods}

2.1. Materials. Dextran Mw of 6000 and 10000, stearoyl chloride, pyrene and dialysis tubing cutoff $2 \mathrm{kDa}$ were purchased from Sigma (USA); dimethyl sulfoxide (DMSO), dimethylformamide (DMF), dimethyl amino pyridine (DMAP), and 3-(4,5-dimethylthiazol-2-yl)-2,5-diphenyl tetrazolium bromide (MTT) were from Merck Chemical Company (Germany). Etoposide was a gift from Nippon Kayaku Co., Ltd. (Tokyo, Japan). Dulbecco's modified Eagle's medium and FBS were supplied from Gibco Laboratories (USA).

2.2. Synthesis of Dextran Stearate. Briefly, $1 \mathrm{~g}$ of different molecular weights (Mws) of dextran (for $\mathrm{Mw}$ of 10000 , $0.1 \mathrm{mmol}$ and for $\mathrm{Mw}$ of $6000,0.166 \mathrm{mmol}$ ) was added to $20 \mathrm{~mL} \mathrm{DMF}$ and stirred at $120^{\circ} \mathrm{C}$ for $2 \mathrm{~h}$ under nitrogen atmosphere. After cooling the slurry to $80^{\circ} \mathrm{C}, 2 \mathrm{~g}$ of LiCl was added and stirred at room temperature for 10 minutes to dissolve completely.

Sufficient amount of DMAP (Table 1) was added to activate the hydroxyl groups of dextran for further nucleophilic attack to carbonyl group of stearoyl chloride. Subsequently, a solution of stearoyl chloride (Table 1) in $20 \mathrm{~mL}$ of DMF was added dropwise in $1 \mathrm{~h}$ at $80^{\circ} \mathrm{C}$ and the mixture was stirred for $24 \mathrm{~h}$.

Ethanol $(200 \mathrm{~mL})$ was added portionwise to the reaction mixture to precipitate the final compound as pale yellow paste which was collected by filtration and washed with ethanol prior to the dryness in vacuum oven [12].

A full factorial design was used for preparation of different copolymers. Two different variables (dextran $\mathrm{Mw}$ and molar ratio of stearate/dextran), each in two levels, were studied as summarized in Table 1. Two digits are seen in the code of each copolymer formulation. The first digit denotes the $\mathrm{Mw}$ of the dextran and the second one the molar ratio of stearate/dextran used in the preparation of each copolymer.

2.3. Determination of Critical Micellar Concentration (CMC). The CMC of the dextran-stearate modified copolymers was obtained by fluorescence spectroscopy using pyrene as a hydrophobic fluorescent probe. Aqueous solutions of decreasing polymer concentrations from $100 \mathrm{mg} / \mathrm{L}$ to $200 \mu \mathrm{g} / \mathrm{L}$ were prepared and mixed with $1 \mathrm{~mL}$ of acetone solution of pyrene $6 \times 10^{-6} \mathrm{~mol} / \mathrm{L}$ while stirred overnight. The final concentration of pyrene was $6 \times 10^{-7} \mathrm{~mol} / \mathrm{L}$.

Measurements of fluorescence intensity were carried out at $\lambda_{\mathrm{em}}=390 \mathrm{~nm}$ and $\lambda_{\mathrm{ex}}=377$ and $338 \mathrm{~nm}$ by spectrofluorimeter (LS-3, Perkin Elmer, USA). The change in ratio of fluorescence intensity $\left(I_{335} / I_{338}\right)$ of pyrene with changes in the concentration of dextran-stearate was recorded for finding the CMC.

2.4. Preparation of Etoposide Loaded Copolymeric Micelles. Etoposide loaded polymeric micelles were prepared by dialysis method. $20 \mathrm{mg}$ of etoposide and $40 \mathrm{mg}$ of copolymer 
were dissolved in $5 \mathrm{~mL}$ of DMSO. The solution was dialyzed against $1 \mathrm{~L}$ of acetate buffer ( $\mathrm{pH} 5.5$ ) for $2 \mathrm{~h}$ in dialysis tubing with cutoff $2 \mathrm{kDa}$, then buffer was exchanged with distilled water and dialysis was continued up to $12 \mathrm{~h}$. The resulting dispersion of micelles was freeze-dried. Blank micelles were prepared by a similar process but without etoposide.

To estimate loading efficacy and loading percent, $5 \mathrm{mg}$ of both blank and drug loaded micelles were dissolved in $10 \mathrm{~mL}$ of DMSO in the volumetric flasks separately and the UV absorptions of resulting solutions were measured in $\lambda_{\max }=$ $284 \mathrm{~nm}$ spectrophotometrically (UV mini 1240, Shimadzu, Japan).

2.5. Size and Zeta Potential of Micelles. Size and zeta potential of micelles were evaluated by dynamic laser light scattering (DLS) method using a nanosizer (Zetasizer ZEN3600, Malvern Instruments Ltd., UK). Five mg of freeze-dried micelles were redispersed in $5 \mathrm{~mL}$ of deionized water and analyzed at a temperature of $25^{\circ} \mathrm{C}$ and a scattering angle of $90^{\circ}$.

2.6. Morphology of Micelles. Atomic force microscopy (AFM) (DME DS 95-50 E, Denmark) was used to study the morphology of the micelles. A drop of micelle dispersion of blank and drug loaded $\mathrm{D}_{6} \mathrm{~S}_{7.5}$ copolymer was applied on mica slides and dried at $40^{\circ} \mathrm{C}$ for $30 \mathrm{~min}$. After the drop was dried, the noncontact mode was used at room temperature.

2.7. In Vitro Drug Release. Five $\mathrm{mg}$ of etoposide loaded polymeric micelles were reconstituted with $1 \mathrm{~mL}$ of phosphate buffered saline (PBS) $(0.1 \mathrm{M}, \mathrm{pH} 7.4)$; then the resulting dispersion was added to dialysis tubing (cutoff $2 \mathrm{kDa}$ ). The dialysis bag was immersed in $50 \mathrm{~mL}$ of PBS and incubated at $37^{\circ} \mathrm{C}$. Samples were withdrawn from the outer fluid at predetermined time intervals up to $48 \mathrm{~h}$. Etoposide concentration was measured spectrophotometrically at $284 \mathrm{~nm}$ and release efficiency of drug was calculated as area under the release curve until $48 \mathrm{~h}$ divided by the total area of $100 \%$ drug released at the same time period.

2.8. Cellular Uptake Studies. Fluorescein loaded micelles were prepared by solubilizing $40 \mathrm{mg}$ of sodium fluorescein and $40 \mathrm{mg}$ of $\mathrm{D}_{6} \mathrm{~S}_{7.5}$ copolymer in $5 \mathrm{~mL}$ of DMSO and then dialyzing the solution against $1 \mathrm{~L}$ of acetate buffer ( $\mathrm{pH}$ 5.5) for $12 \mathrm{~h}$ in dialysis tubing with cutoff $2 \mathrm{kDa}$. The buffer was then exchanged with distilled water and dialysis was continued for $24 \mathrm{~h}$. The resulting dispersion was freeze-dried.

A sterile glass cover was used in each well of a 6-well culture plate. $1 \times 10^{5}$ cells of CT- 26 cell line were seeded into each well and incubated for $24 \mathrm{~h}$ at $37^{\circ} \mathrm{C} .100 \mu \mathrm{g}$ of sodium fluorescein or $200 \mu \mathrm{g}$ of fluorescein loaded micelles was added to each well. After 4 - and 24 -h incubation at $37^{\circ} \mathrm{C}$, the cells were washed three times with PBS. A fluorescent microscope (CETI, Belgium) was used for cell imaging.

2.9. In Vitro Cytotoxicity Test of Etoposide Loaded Micelles. In vitro cytotoxicity of etoposide loaded micelles was determined by MTT assay. CT-26 cells were washed in exponential growth phase, trypsinized, and resuspended in DMEM culture medium. Then dilution of cell suspension was carried out to obtain $10^{4}$ cells $/ \mathrm{mL}$. $180 \mu \mathrm{L}$ of this suspension was poured to each well of a 96-well plate and incubated in a humidified incubator in an atmosphere of $5 \% \mathrm{CO}_{2}$ for $24 \mathrm{~h}$ until a partial monolayer was formed. Then the cells were exposed to various concentrations of the blank and drug loaded micelles and free drug in quadruplicate. After $48 \mathrm{~h}$ incubation, the cells were exposed to MTT solution $5 \mathrm{mg} / \mathrm{mL}$ for $3 \mathrm{~h}$. When insoluble formazan crystals were formed, the supernatant fluid was removed and crystals were solubilized with $150 \mu \mathrm{L}$ of DMSO. The absorbance of each sample was measured at $570 \mathrm{~nm}$ using an automated microplate reader (Awareness, USA).

\section{Results and Discussion}

3.1. Synthesis and Characterization of Dextran Stearate Copolymer. Dextran stearate was synthesized by acylation of dextran with stearoyl chloride. Du et al. [9] performed this synthesis via carbodiimide reaction, but acylation method used in our study by acyl chloride is cheaper and the purification of final product is easier and faster. Instrumental analysis of final compound confirmed this graft.

The reaction between stearoyl chloride and dextran was confirmed in ${ }^{1} \mathrm{H}$ NMR spectrum by two confirmative signals related to $\mathrm{CH}_{2} \mathrm{CH}_{2}$ next to the carbonyl group of stearoyl chloride (Figure 1). In case of failure in synthesis of desired compound, stearoyl chloride will turn to the stearic acid in reaction condition and these signals should be observed at 1.46 and $2.18 \mathrm{ppm}$ as is seen in standard spectra of stearic acid (Figure 1(a)). Successful esterification of dextran will move these signals to higher domains ( $\delta \mathrm{ppm})$ due to the deshielding effect of ester bond formation on adjuvant methylene groups in the product. The signals of these protons are shifted slightly downfield as is expected and are observed at 1.50 and $2.31 \mathrm{ppm}$, respectively, in final compound spectra (Figure 1(c)).

In the IR spectrum, the signal related to the stretching vibration of the carbonyl group of stearic acid was observed at $1705 \mathrm{~cm}^{-1}$ (Figure 2(a)). This signal is shifted to $1747 \mathrm{~cm}^{-1}$ in product (Figure 2(b)), which is due to the $\mathrm{C}=\mathrm{O}$ stretching vibration of ester group formed. So it can be concluded that acid is converted to ester in product.

One of the most useful and accurate procedure for calculation of degree of substitution (DS) in polymer synthesis is application of ${ }^{1} \mathrm{H}$ NMR spectra. In this method, at least one signal related to each part of the synthesized polymer from two subunits should be nominated as representative of each compound.

Signals should be clearly distinguishable from the rest of the spectra without any overlapping or interfering. Area under the peak of these signals should be correlated with their number of the hydrogen atoms. DS is simply calculated from mathematical calculations based on comparison between integrals of these signals $[9,12]$.

In this study, the end methyl group of stearate $(\delta=$ $0.84933 \mathrm{ppm}$ ) is well upfield comparing to the other signals 


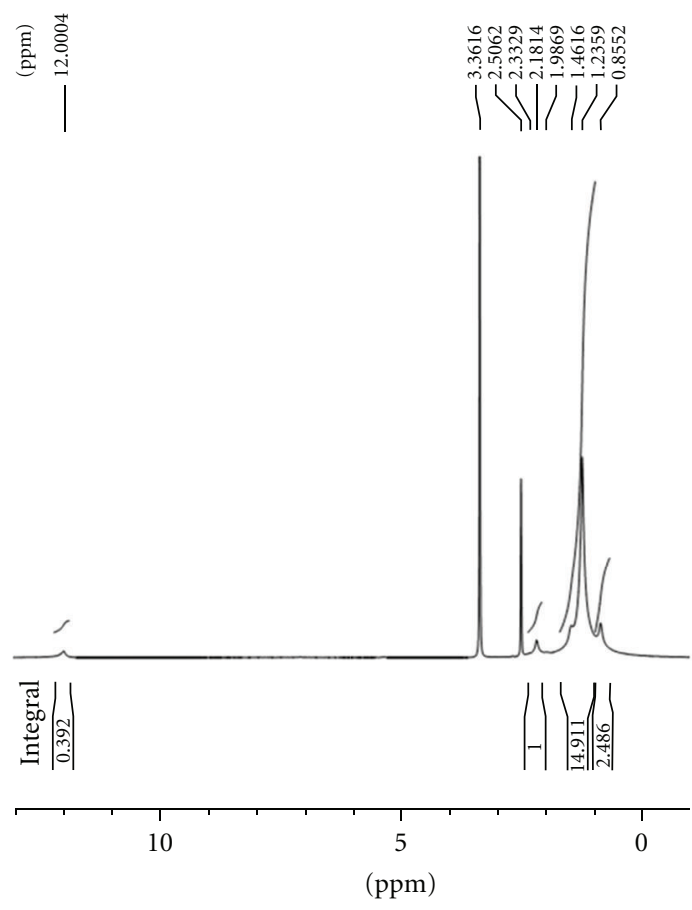

(a)

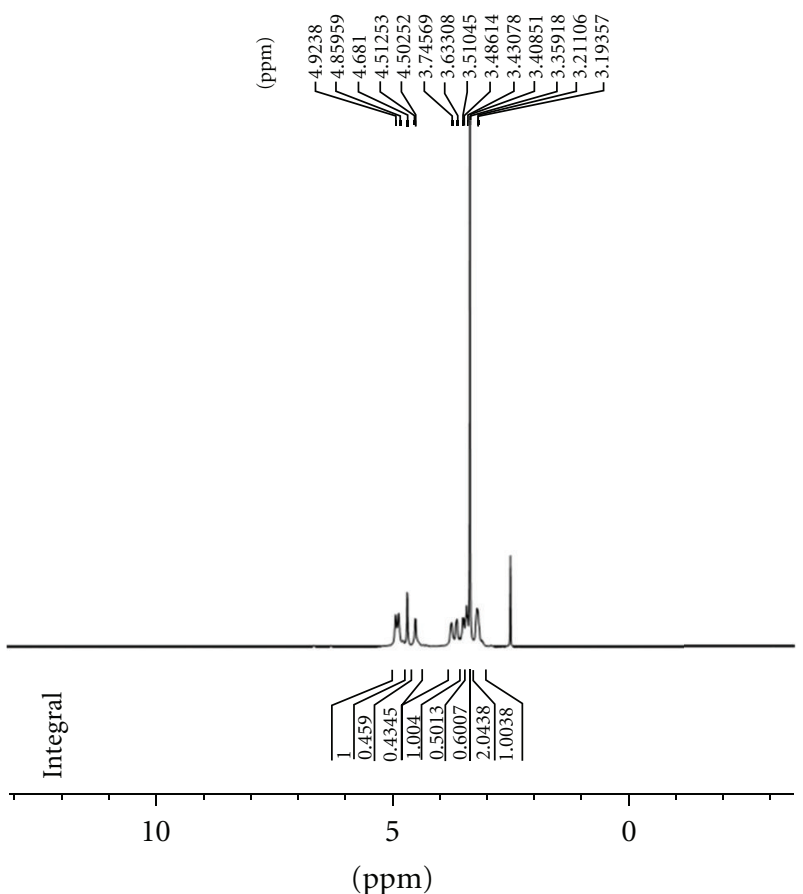

(b)

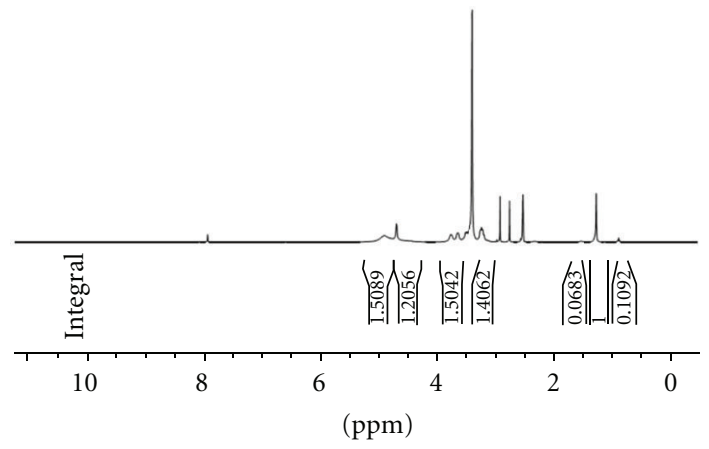

(c)

FIGURE 1: ${ }^{1} \mathrm{H}$ NMR spectra of (a) stearic acid, (b) dextran 6000 , and (c) $\mathrm{D}_{6} \mathrm{~S}_{7.5}$ copolymer.

due to the higher shielding effects of neighboring methylene groups (Figure 1(a)). The anomeric proton of dextran is also clearly distant from other signals $(\delta=4.84976 \mathrm{ppm})$ (Figure 1(b)). These signals were chosen for mathematical calculations. Table 2 represents DS of different prepared copolymers.

3.2. CMC of Dextran Stearate Copolymers. There are different methods for estimation of CMC. One of the most recurrent methods is using vibronic band intensity changing in pyrene's fluorescence due to passing the pyrene from a hydrophilic to a hydrophobic environment [13]. Normally emission spectra of pyrene strongly depend on polarity of microenvironment; in polar solvent, intensity of the first energy band $\left(I_{1}\right)$ is higher than the third energy band $\left(I_{3}\right)$ of the pyrene emission spectra while in hydrophobic environment, it is vice versa, that is, the intensity of $I_{3}$ is higher than that of $I_{1}$. Therefore, when micelles are formed in aqueous medium, the pyrene is preferably partitioned into hydrophobic core and intensity of $I_{3}$ increases. As a result, the ratio of $I_{1} / I_{3}$ can be used to determine CMC. Recently, more accurate results have been achieved by monitoring changes 


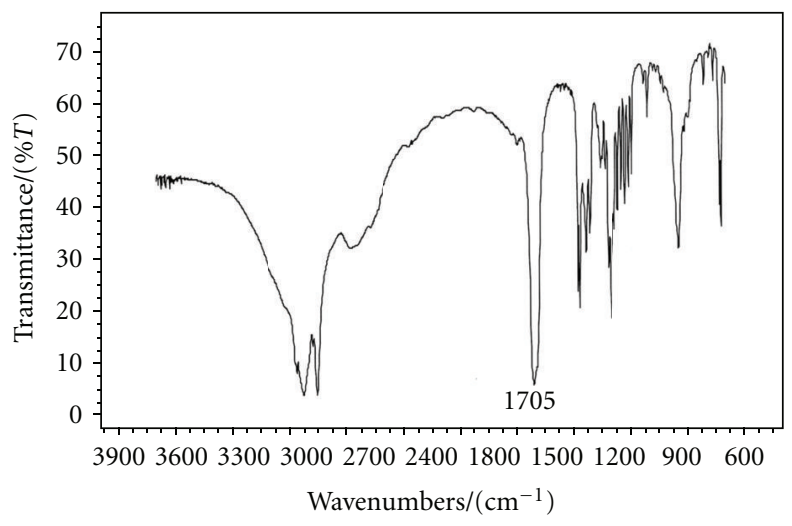

(a)

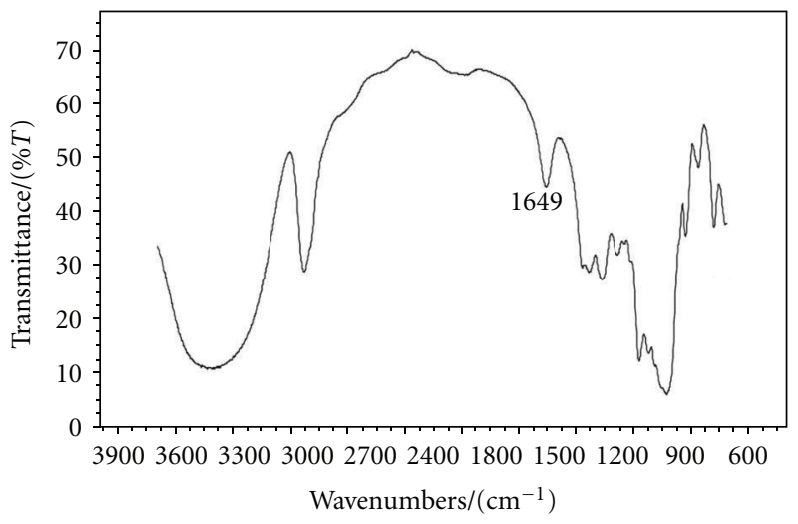

(b)

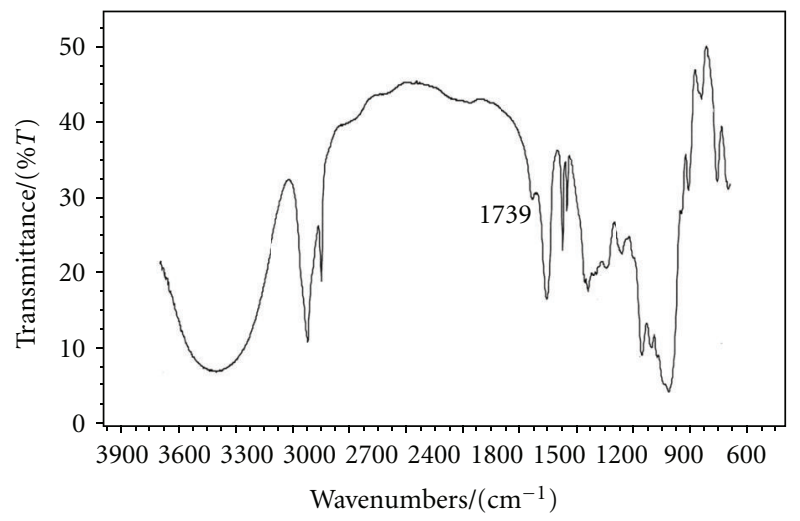

(c)

FIGURE 2: FTIR spectra of (a) stearic acid, (b) dextran 6000, and (c) $\mathrm{D}_{6} \mathrm{~S}_{7.5}$ copolymer.

in the ratio of the pyrene's excitation intensity spectra [14]. Here also in our study we used a fixed-emission wave length of $\lambda_{\mathrm{em}}=390 \mathrm{~nm}$ and the excitation intensities were obtained for pyrene.

Maximum fluorescence intensity of $I_{3}$ for pyrene in water was $\lambda_{\mathrm{ex}}=335 \mathrm{~nm}$ and for pyrene in a hydrophobic medium (in presence of dextran stearate copolymers) was $\lambda_{\mathrm{ex}}=$ $338 \mathrm{~nm}$ (Figure 3).

The ratio of $I_{338} / I_{335}$ versus different concentrations of dextran stearate copolymers in aqueous solutions is shown in Figure 4.

The CMC was determined from the intersection of two straight lines. The CMC values for each modified dextran are represented in Table 2. The results show considering low $\mathrm{CMC}$ values obtained for the dextran stearate copolymers compared to the high values of surfactants they can form stable micelles in aqueous solutions. The CMC values obtained for dextran copolymers (Table 2) show that they are about 100-300 times lower than that of low-molecularmass surfactant micelles like sodium dodecyl sulphate with $\mathrm{CMC}$ of $2.3 \mathrm{mg} \mathrm{mL}^{-1}$ [15]. It means that these copolymers can form more thermodynamically stable micelles under highly diluted conditions. The results of statistical analysis of $\mathrm{CMC}$ data also indicate that by increasing molecular weight of dextran and stearoyl chloride molar ratio, the CMC was increased in resulting polymers $(P<0.05)$.
3.3. Physical Characteristics and Drug Loading Efficiency of Dextran Stearate Micelles. Particle size, zeta potential, and etoposide loading efficiency in different drug loaded copolymer micelles are shown in Table 3. Etoposide was loaded in micelles by dialysis method. This method can make uniform micelles without additional free drug. Also for such polymers that do not have enough solubility in organic solvents, dialysis method will be an unavoidable choice [16].

Size of the micelles increased in higher DS (Table 2), but polymer molecular weight had no significant effect on particle size of unloaded micelles $(P>0.05)$. Recently Acharya and Sahoo [17] have shown that for successful targeting of PLGA nanoparticles through EPR effect the particle size below $200 \mathrm{~nm}$ is needed. Such particles can pass through fenestrated tumor capillaries with minimal absorption in reticuloendothelial system. In the present study, formulations of $D_{6} S_{7.5}$ and $D_{10} S_{7.5}$ had been adaptable for these purposes theoretically (Table 2).

Zeta potential is a good measure for stability of micelles versus aggregation. Decreasing the zeta potential of micelles after drug loading in all formulations means that stability of micelles is weakened. Maximum absolute value of zeta potential was pertinent to $D_{6} S_{7.5}$ formulation, and consequently it may conclude that it was the most stable formulation.

The copolymer of $D_{6} S_{7.5}$ showed encapsulation efficiency of about $100 \%$ for etoposide, while drug loading percent 
Table 2: Physicochemical properties of different synthesized copolymers.

\begin{tabular}{lccccc}
\hline Dextran stearate copolymer & DS $(\%)$ & Particle size $(\mathrm{nm})$ & Polydisparity index & Zeta potential $(\mathrm{mV})$ & CMC mg/L \\
\hline $\mathrm{D}_{6} \mathrm{~S}_{7.5}$ & 2.41 & $131.7 \pm 2.5$ & $0.46 \pm 0.02$ & -30.2 & 7.44 \\
$\mathrm{D}_{6} \mathrm{~S}_{10}$ & 2.99 & $293 \pm 0.2$ & $0.35 \pm 0.01$ & -23.9 & 8.93 \\
$\mathrm{D}_{10} \mathrm{~S}_{7.5}$ & 2.37 & $133 \pm 9.5$ & $0.33 \pm 0.11$ & -22.3 & 14.98 \\
$\mathrm{D}_{10} \mathrm{~S}_{10}$ & 3.40 & $194.8 \pm 17.8$ & $0.31 \pm 0.04$ & -12.3 & 22.08 \\
\hline
\end{tabular}

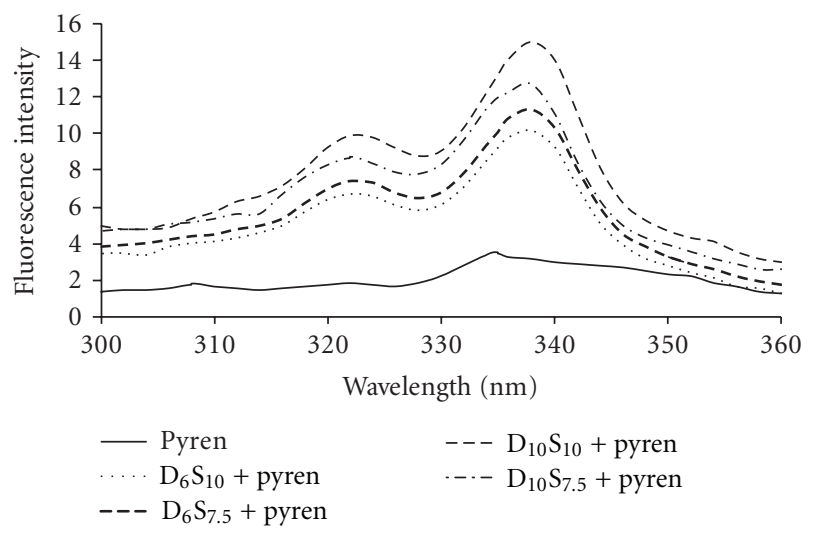

FIGURE 3: Excitation spectra of pyrene in aqueous solution monitored at $\lambda_{\text {em }}$ of $390 \mathrm{~nm}$ in absence or presence of different dextran stearate copolymers at a concentration of $25 \mathrm{mg} / \mathrm{L}$.

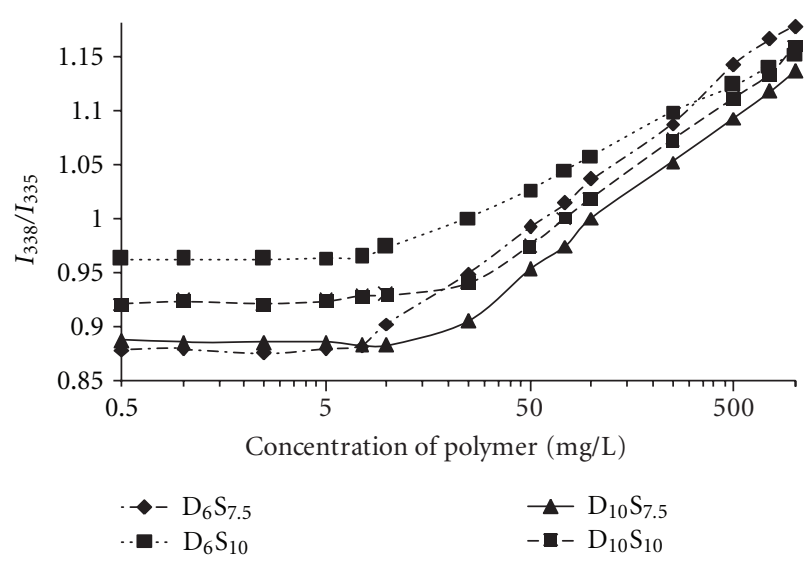

Figure 4: Changes of fluorescence intensity ratio $\left(I_{338} / I_{335}\right)$ versus $\log$ of copolymers concentration.

was around 33\% according to total dried copolymer weight (Table 3).

In the previous study on dextran stearate [9], molecular weights of $10000 \mathrm{kDa}$ and higher had been tested, and the authors concluded that $10000 \mathrm{kDa}$ was the optimum molecular weight for delivery of doxorubicin, but in the present study the molecular weight of $6000 \mathrm{kDa}$ has shown better results in vitro. Increasing in dextran molecular weight and molar ratio of stearoyl chloride had reverse effect on loading efficiency of etoposide in dextran stearate micelles $(P<0.05)$.

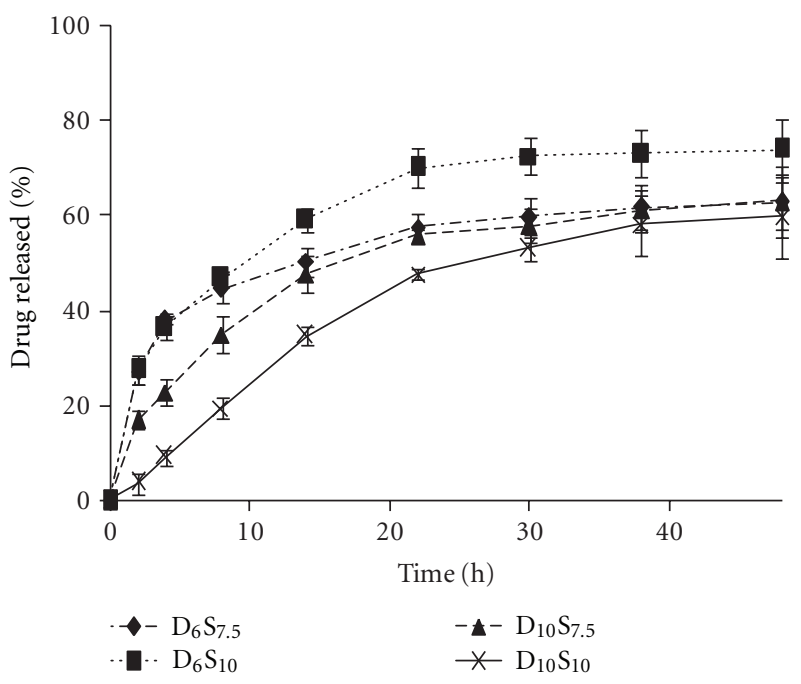

FIGURE 5: Etoposide release profiles from dextran stearate polymeric micelles in phosphate buffer saline $\mathrm{pH} 7.4(n=3)$.

3.4. In Vitro Drug Release. Release tests were performed in phosphate buffered saline (PBS; 0.1 M, pH 7.4), and Figure 5 represents release profiles of etoposide from different formulation. $\mathrm{D}_{10} \mathrm{~S}_{10}$ was the most insoluble modified dextran and had the slowest release profile. Etoposide release from this polymer showed near-zero-order kinetic and hence maybe suitable for sustained release drug delivery. Other formulations exhibited sustained release properties followed by a minor initial burst release. Prolonged release can be attributed to the slow diffusion of drug from the micelles.

Release profile of etoposide from $D_{10} S_{7.5}$ and $D_{6} S_{7.5}$ is similar except for the initial burst release of drug from $\mathrm{D}_{6} \mathrm{~S}_{7.5}$ copolymer. Drug release rate and amount from $\mathrm{D}_{6} \mathrm{~S}_{10}$ had been the greatest. Drug release efficiency of micelles is shown in Table 3. Statistical analysis showed that increasing in polymer molecular weight had reverse effect on $\mathrm{RE}_{48} \%$, that is, increasing the Mw of dextran decreased the drug release efficiency from the micelles. However, effect of stearoyl chloride molar ratio was more complicated. For modified dextran with molecular weight of 10000, increasing the molar ratio of stearate decreased the $\mathrm{RE}_{48} \%$ but for copolymers with dextran molecular weight of 6000 a reverse effect was seen and higher molar ratio of stearate enhanced the $\mathrm{RE}_{48} \%$ significantly $(P<0.05)$.

3.5. Atomic Force Microscopy. The morphology of micelles was observed by atomic force microscopy (AFM). Two- 

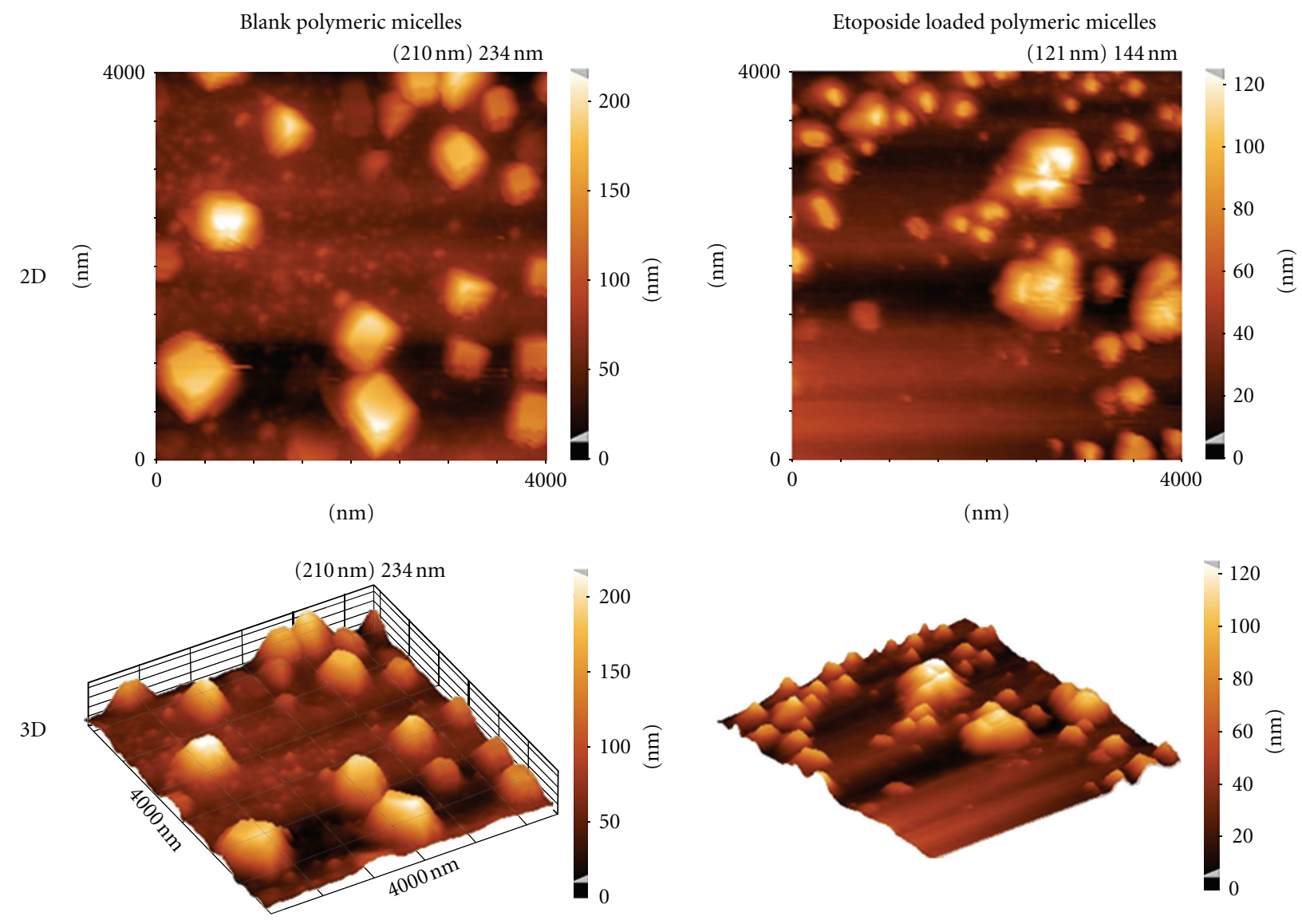

Figure 6: Two- and three-dimensional AFM imaging of blank and drug loaded micelles of dextran-stearate.

TABLE 3: Physical characteristics of etoposide-loaded dextran stearate micelles.

\begin{tabular}{lcccccc}
\hline $\begin{array}{l}\text { Dextran stearate } \\
\text { copolymer }\end{array}$ & $\begin{array}{c}\text { Particle size } \\
(\mathrm{nm})\end{array}$ & Polydisparity index & $\begin{array}{c}\text { Zeta potential } \\
\mathrm{mV}\end{array}$ & $\begin{array}{c}\text { Drug loading \% } \\
\text { (169) }\end{array}$ & $\begin{array}{c}\text { Drug loading } \\
\text { efficiency \% }\end{array}$ & $\begin{array}{c}\text { Release efficiency } \\
\left(\mathrm{RE}_{48} \%\right)\end{array}$ \\
\hline $\mathrm{D}_{6} \mathrm{~S}_{7.5}$ & $294.7 \pm 11.1$ & $0.363 \pm 0.002$ & -19.2 & $31.7 \pm 0.8$ & $93.3 \pm 2.4$ & $51.15 \pm 1.32$ \\
$\mathrm{D}_{6} \mathrm{~S}_{10}$ & $175.8 \pm 6.7$ & $0.374 \pm 0.025$ & -11.51 & $30.3 \pm 0.1$ & $90.5 \pm 0.5$ & $62.14 \pm 1.19$ \\
$\mathrm{D}_{10} \mathrm{~S}_{7.5}$ & $284.3 \pm 29.2$ & $0.346 \pm 0.013$ & -7.23 & $18.5 \pm 0.1$ & $55.7 \pm 0.2$ & $48.68 \pm 2.73$ \\
$\mathrm{D}_{10} \mathrm{~S}_{10}$ & & & & $15.1 \pm 1.6$ & $45.2 \pm 4.9$ & $40.81 \pm 0.35$ \\
\hline
\end{tabular}

and three-dimensional AFM imaging of blank and drug loaded $\mathrm{D}_{6} \mathrm{~S}_{7.5}$ polymeric micelles is shown in Figure 6. In the images of blank micelles cubic-shaped aggregates and finer spherical micelles are seen. But in drug loaded micelles the aggregates were less and shapeless. Micelles have oval and spherical shape in this case. AFM gives an overestimation of particle size as a result of the AFM tip-broadening effect. The magnitude of the tip dilation effect depends on the height of the object, the ambient humidity, and the size and shape of the AFM tip. There are evidences that confirm hydrophilic interactions between polymeric micelles and surface of mica. These interactions cause micelles to spread on the surface of mica $[18,19]$.

3.6. Cellular Uptake Studies. The cellular uptake tests on CT26 murine colorectal adenocarcinoma cells were performed using sodium fluorescein-labeled $\mathrm{D}_{6} \mathrm{~S}_{7.5}$ copolymer and solution of sodium fluorescein 4 and $24 \mathrm{~h}$ after incubation with the cells. The micrographs were taken using visible or fluorescent light. The results are represented in Figure 7.

As this figure shows, after $4 \mathrm{~h}$ the green fluorescence is sighted in cells that are incubated with sodium fluoresceinloaded $\mathrm{D}_{6} \mathrm{~S}_{7.5}$ micelles, but there is no significant fluorescence in cells that are incubated with solution of sodium fluorescein. After $24 \mathrm{~h}$, green fluorescence was visible in both fluorescein-loaded micelles and solution of sodium fluorescein incubated cells. The accumulation of sodium fluorescein after $24 \mathrm{~h}$ may be due to proton concentration changes in the medium caused by glycolytic respiration of the cells [20].

3.7. In Vitro Cytotoxicity. In order to compare the cytotoxicity of different formulations, pure polymers and etoposide 

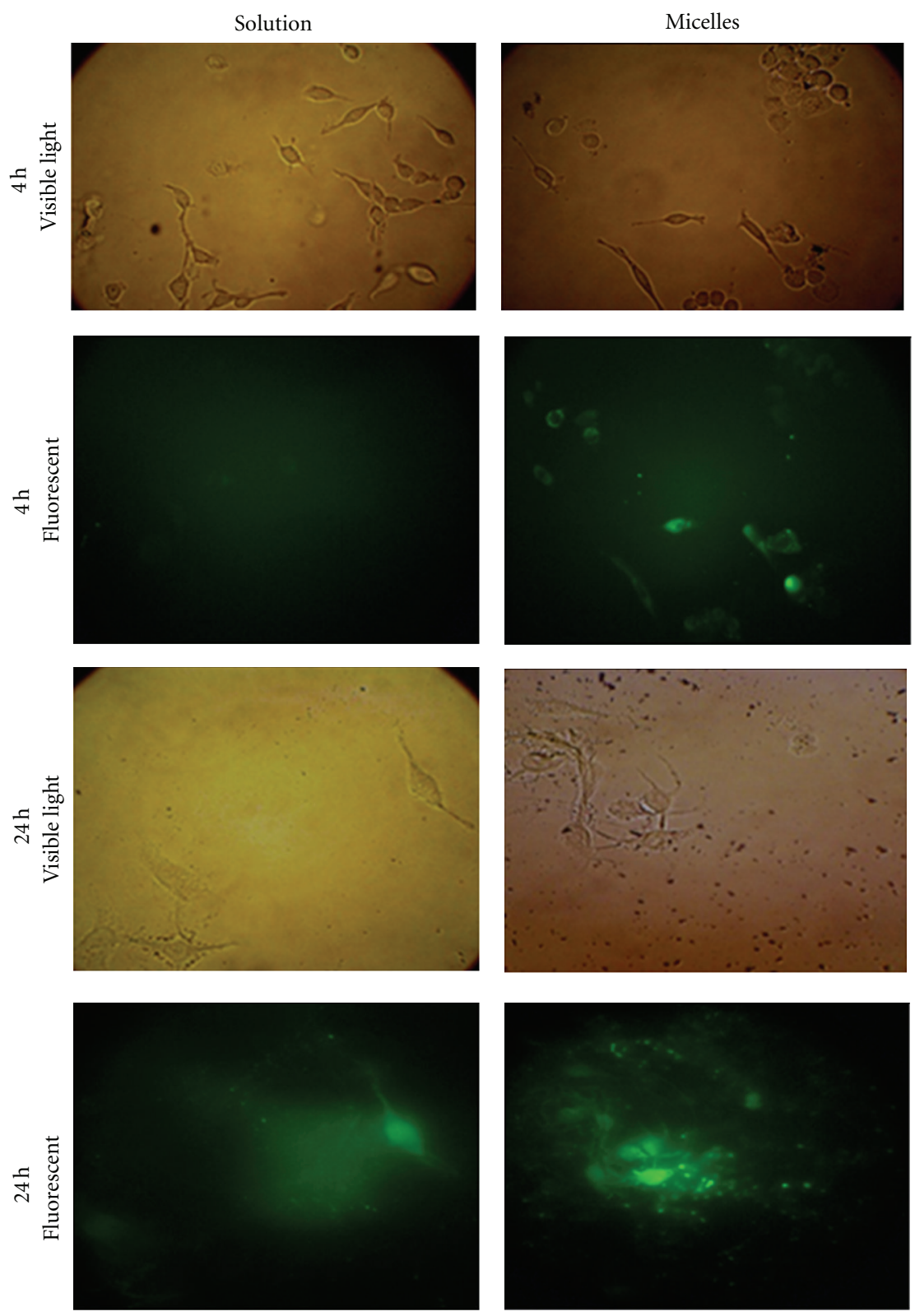

Figure 7: Fluorescent and visible light microscopic images of CT26 cells after incubation with fluorescein loaded micelles or fluorescein solution for 4 and $24 \mathrm{~h}$.

in CT-26 adenocarcinoma cell line, $\mathrm{IC}_{50}$ was calculated from MTT assay data (Figure 8).

As this figure indicates, the $\mathrm{IC}_{50}$ of pure etoposide in DMSO $1 \% \mathrm{v} / \mathrm{v}$ was $9.1 \pm 0.64 \mu \mathrm{g} / \mathrm{mL}$ while that of the pure copolymers was greater and all drug loaded micelles showed significantly lower $\mathrm{IC}_{50}$ than pure drug $(P<0.05)$. Dextran micelles loaded with etoposide and containing lower stearate content were more cytotoxic. Statistical analysis showed that the molar ratio of stearate had a significant effect on $\mathrm{IC}_{50}$ of etoposide loaded micelles, but polymer molecular weight had no significant effect on $\mathrm{IC}_{50}$ of etoposide loaded in micelles. Changes in molar ratio and molecular weight had significant effect on $\mathrm{IC}_{50}$ of blank micelles $(P<0.05)$.
Etoposide loaded in $\mathrm{D}_{6} \mathrm{~S}_{7.5}$ and $\mathrm{D}_{10} \mathrm{~S}_{7.5}$ copolymers was more cytotoxic than other copolymers. It is notable that these micelles had almost similar drug release profiles. The results of release tests (Figure 5) showed that only $70 \%$ to $80 \%$ of the total loaded drug in micelles can be released within $48 \mathrm{~h}$, but it seems that this amount had greater toxic effect than pure drug. An explanation for this observation may be interpreted so that colorectal cancer cell are resistant to etoposide and one solution for overcoming multidrug resistance is using colloidal drug delivery systems. This strategy is based on estimated uptake of the nanocarriers into the cancerous cells by endocytosis. Under these conditions, the concentration of the absorbed drug will be more than the excreted amount by the expelling pumps and enzymes [21]. 


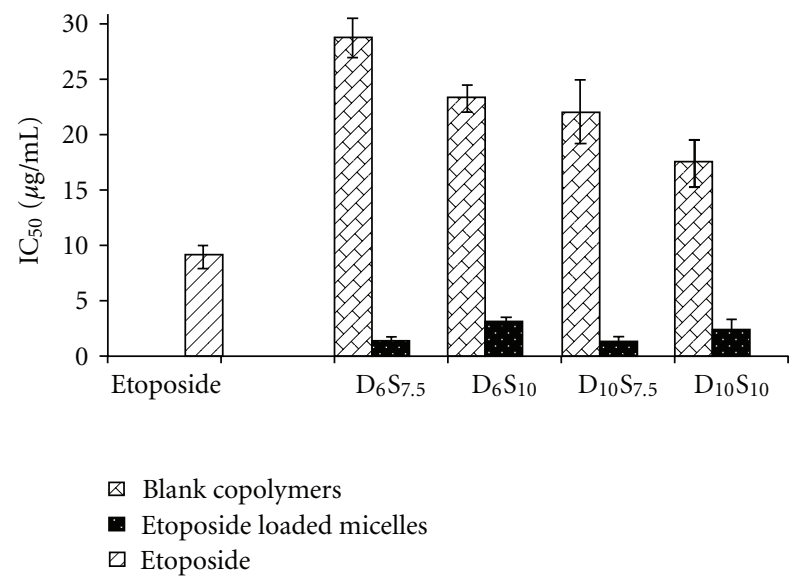

Figure 8: IC $_{50}$ of etoposide, blank copolymers of dextran stearate, and the etoposide loaded micelles.

Necessarily, the release rate of drug from colloidal carriers should be not too fast, which causes total drug release before endocytosis of the particles and not too slow that the resistance mechanism can overcome this augmented intracellular concentration.

\section{Conclusions}

Dextran was modified with stearic acid substitution successfully by an easier and faster method than previously reported method. The resulting amphiphilic copolymers had low CMC. Drug loaded copolymeric micelles could release etoposide more than $48 \mathrm{~h}$. The micelles had good cellular uptake capability and etoposide loaded micelles were more cytotoxic than those of free drug in CT-26 cell line. So formulation of etoposide in dextran stearate micelles may be a practical solution for conquest on drug resistance in colorectal cancer. Degree of substitution of polymer with stearic acid affected drug loading, release rate, particle size, and cytotoxicity of the blank and etoposide loaded micelles. Although molecular weight of dextran did not have any effective role on particle size and cytotoxicity of the etoposide loaded micelles, it showed significant effect on drug loading efficiency, drug release rate, CMC, and zeta potential of micelles that all affect the stability of drug formulation.

\section{Acknowledgment}

Foundation support of this study by the Research Vice Chancellor of Isfahan University of Medical Sciences is acknowledged.

\section{References}

[1] M. Bikram, C. H. Ahn, S. Y. Chae, M. Lee, J. W. Yockman, and S. W. Kim, "Biodegradable poly(ethylene glycol)-co-poly(llysine)-g-histidine multiblock copolymers for nonviral gene delivery," Macromolecules, vol. 37, no. 5, pp. 1903-1916, 2004.
[2] V. S. Trubetskoy, "Polymeric micelles as carriers of diagnostic agents," Advanced Drug Delivery Reviews, vol. 37, no. 1-3, pp. 81-88, 1999.

[3] A. Mahmud, X. B. Xiong, H. M. Aliabadi, and A. Lavasanifar, "Polymeric micelles for drug targeting," Journal of Drug Targeting, vol. 15, no. 9, pp. 553-584, 2007.

[4] H. Maeda, "The enhanced permeability and retention (EPR) effect in tumor vasculature: the key role of tumor-selective macromolecular drug targeting," Advances in Enzyme Regulation, vol. 41, pp. 189-207, 2001.

[5] T. Minko, P. Kopečkova, V. Pozharov, K. D. Jensen, and J. Kopeček, "The influence of cytotoxicity of macromolecules and of VEGF gene modulated vascular permeability on the enhanced permeability and retention effect in resistant solid tumors," Pharmaceutical Research, vol. 17, no. 5, pp. 505-514, 2000.

[6] S. K. Hobbs, W. L. Monsky, F. Yuan et al., "Regulation of transport pathways in tumor vessels: role of tumor type and microenvironment," Proceedings of the National Academy of Sciences of the United States of America, vol. 95, no. 8, pp. 46074612, 1998.

[7] S. S. Dhaneshwar, M. Kandpal, N. Gairola, and S. S. Kadam, "Dextran: a promising macromolecular drug carrier," Indian Journal of Pharmaceutical Sciences, vol. 68, no. 6, pp. 705-714, 2006.

[8] R. J. Mumper and A. S. Hoffman, "The stabilization and release of hirudin from liposomes or lipid-assemblies coated with hydrophobically modified dextran," AAPS PharmSciTech, vol. 1, no. 1, article 3, 2000.

[9] Y. Z. Du, Q. Weng, H. Yuan, and F. Q. Hu, "Synthesis and antitumor activity of stearate-g-dextran micelles for intracellular doxorubicin delivery," ACS Nano, vol. 4, no. 11, pp. 6894-6902, 2010.

[10] J. P. Braybrooke, N. C. Levitt, S. Joel et al., "Pharmacokinetic study of cisplatin and infusional etoposide phosphate in advanced breast cancer with correlation of response to topoisomerase II $\alpha$ expression," Clinical Cancer Research, vol. 9, no. 13, pp. 4682-4688, 2003.

[11] H. S. Na, Y. K. Lim, Y. I. Jeong et al., "Combination antitumor effects of micelle-loaded anticancer drugs in a CT26 murine colorectal carcinoma model," International Journal of Pharmaceutics, vol. 383, no. 1-2, pp. 192-200, 2010.

[12] M. A. Hussain, Alternative Routes of Polysaccharide Acylation: Synthesis, Structural Analysis. Properties, Jena: FriedrichSchiller-University, 2004.

[13] C. L. Zhao, M. A. Winnik, G. Riess, and M. D. Croucher, "Fluorescence probe techniques used to study micelle formation in water-soluble block copolymers," Langmuir, vol. 6, no. 2, pp. 514-516, 1990.

[14] M. F. Francis, L. Lavoie, F. M. Winnik, and J. C. Leroux, "Solubilization of cyclosporin A in dextran-g-polyethyleneglycolalkyl ether polymeric micelles," European Journal of Pharmaceutics and Biopharmaceutics, vol. 56, no. 3, pp. 337346, 2003.

[15] F. Bian, L. Jia, W. Yu, and M. Liu, "Self-assembled micelles of N-phthaloylchitosan-g-polyvinylpyrrolidone for drug delivery," Carbohydrate Polymers, vol. 76, no. 3, pp. 454-459, 2009.

[16] L. Liang, J. Ping, C. Ming, Z. Guoliang, and Z. Fengbao, "5fluorouracil-loaded self-assembled $\mathrm{pH}$-sensitive nanoparticles as novel drug carrier for treatment of malignant tumors," Chinese Journal of Chemical Engineering, vol. 14, no. 3, pp. 377382, 2006.

[17] S. Acharya and S. K. Sahoo, "PLGA nanoparticles containing various anticancer agents and tumour delivery by EPR effect," 
Advanced Drug Delivery Reviews, vol. 63, no. 3, pp. 170-183, 2011.

[18] K. B. Thurmond II, H. Huang, C. G. Clark, T. Kowalewski, and K. L. Wooley, "Shell cross-linked polymer micelles: stabilized assemblies with great versatility and potential," Colloids and Surfaces B, vol. 16, no. 1-4, pp. 45-54, 1999.

[19] J. Liaw, T. Aoyagi, K. Kataoka, Y. Sakurai, and T. Okano, "Visualization of PEO-PBLA-pyrene polymeric micelles by atomic force microscopy," Pharmaceutical Research, vol. 15, no. 11, pp. 1721-1726, 1998.

[20] O. V. Braginskaja, V. V. Lazarev, I. N. Pershina, K. V. Petrov, L. B. Rubin, and O. V. Tikhonova, "Sodium fluorescein accumulation in cultured cells," General Physiology and Biophysics, vol. 12, no. 5, pp. 453-464, 1993.

[21] A. Lamprecht and J. P. Benoit, "Etoposide nanocarriers suppress glioma cell growth by intracellular drug delivery and simultaneous P-glycoprotein inhibition," Journal of Controlled Release, vol. 112, no. 2, pp. 208-213, 2006. 

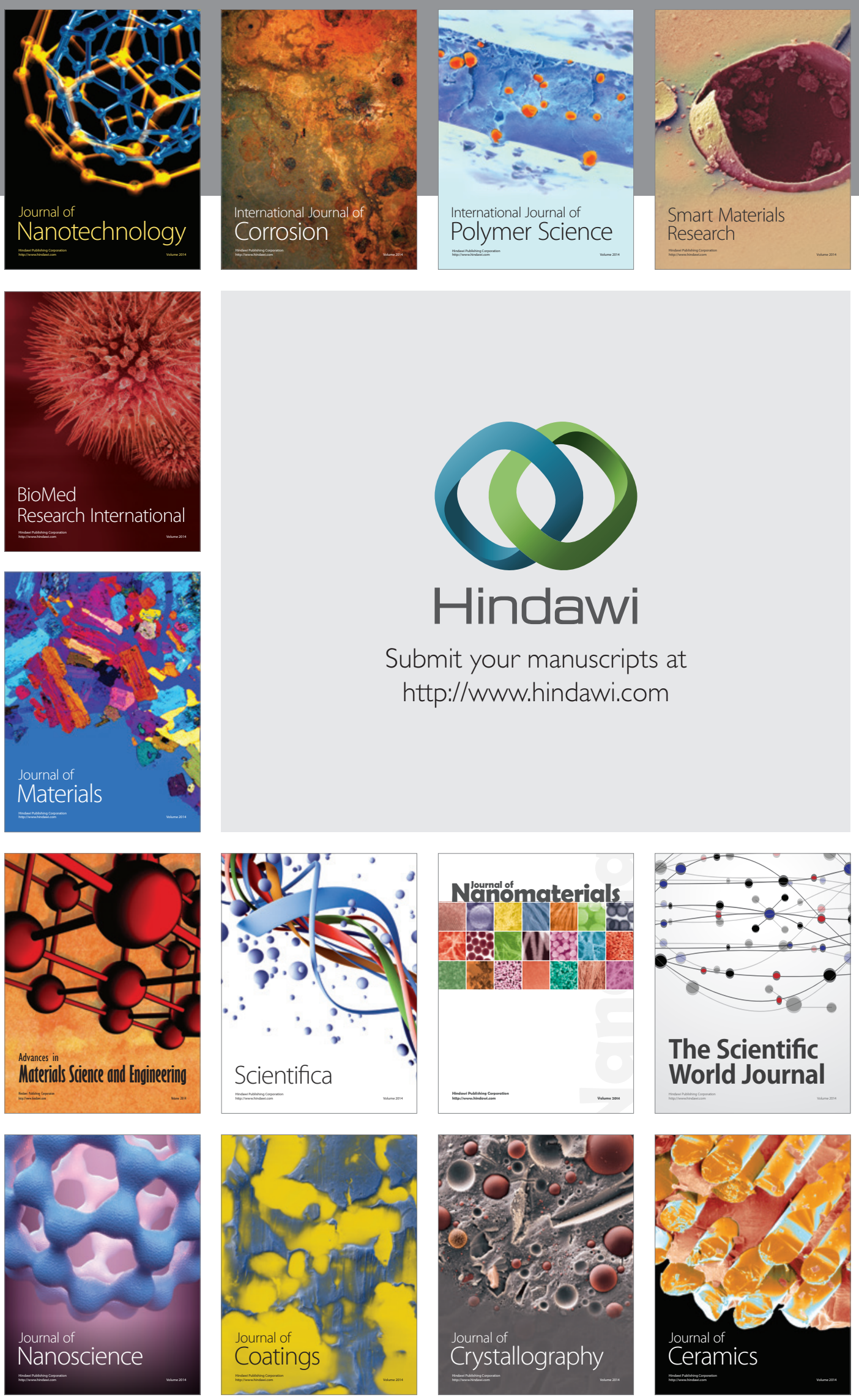

The Scientific World Journal

Submit your manuscripts at

http://www.hindawi.com

\section{World Journal}

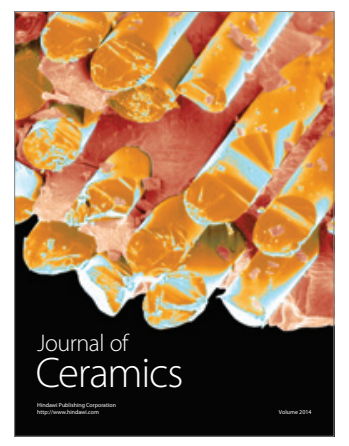

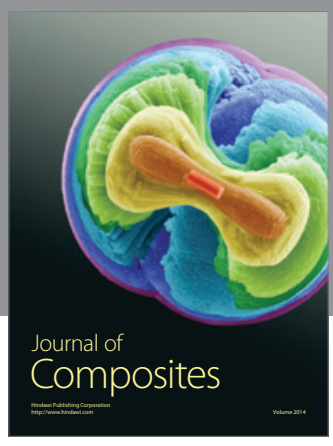
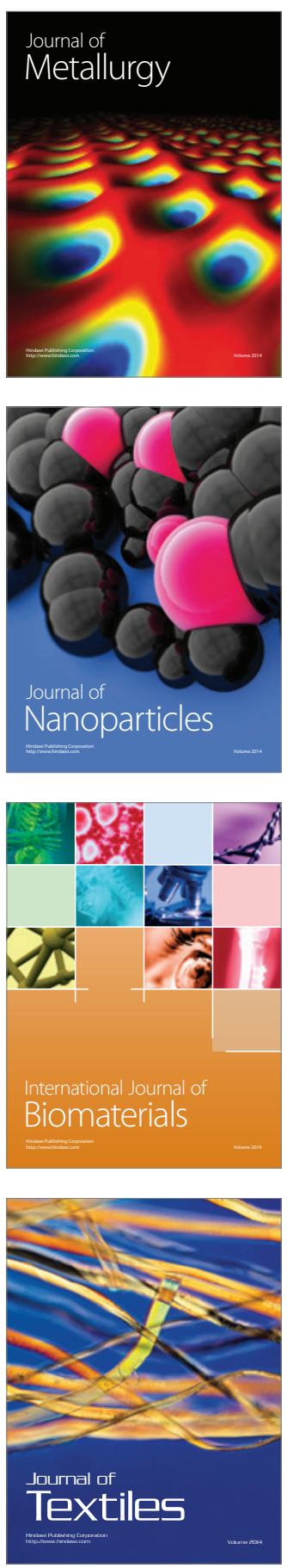